\title{
A Flexible Approach to Modelling Adaptive Course Sequencing based on Graphs implemented using XLink
}

\author{
Rachid ELOUAHBI \\ Department of mathematics and computer Science, Moulay \\ Ismail University, Faculty of Sciences Meknes, Morocco
}

\section{Noreddine ABGHOUR}

Department of mathematics and computer Science, Hassan

II Ain Chock University,Faculty of Sciences, Casablanca, Morocco

\begin{abstract}
Driss BOUZIDI
Department of mathematics and computer Science, Hassan II Ain Chock University,Faculty of Sciences, Casablanca, Morocco

Mohammed Adil NASSIR

Department of mathematics and computer Science, Moulay Ismail University, Faculty of Sciences Meknes,Morocco
\end{abstract}

\begin{abstract}
A major challenge in developing systems of distance learning is the ability to adapt learning to individual users. This adaptation requires a flexible scheme for sequencing the material to teach diverse learners. This is where we intend to contribute to model the personalized learning paths to be followed by the learner to achieve his/her determined educational objective. Our modelling approach of sequencing is based on the pedagogical graph which is called SMARTGraph. This graph allows expressing the totality of the pedagogic constraints under which the learner is submitted in order to achieve his/her pedagogic objective. SMARTGraph is a graph in which the nodes are the learning units and the arcs are the pedagogic constraints between learning units.

We shall see how it is possible to organize the learning units and the learning paths to answer the expectations within the framework of individual courses according to the learner profile or within the framework of group courses. To implement our approach we exploit the strength of XLink (XML Linking Language) to define the sequencing graph.
\end{abstract}

Keywords- e-learning; pedagogical graph; adaptability; profile; learning units; XML; XLINK; XSL.

\section{INTRODUCTION AND STATE OF THE ART}

In the e-Learning context, the sequencing means the scheduling, planning or also the organization of the elements constituting the content to be taught. In order to carry out the pedagogic sequencing, it is necessary to know the learners profile and the objectives of the training. From a given detailed representation of the contents, the sequencing authorizes a dynamic planning of the course elements that will be the subject of a pedagogic activity. This representation describes in a structured and hierarchical way the scheduling of the objectives to be achieved in order to meet the terminal goals as fixed by the course.

It is very important to design the course sequencing, since it leaves the traditional relation between teacher and learners [6]. It is necessary for us to exploit various contexts so that the trainings are built through various interactions. In the context of distance learning, there is no more exposure of knowledge, and declaratory knowledge assimilation by learners, who will thereafter be implemented in evaluation situations, and translated into competency. It is rather necessary to offer the learner an interactive framework which will not only guide his/her course in the sequences of teaching, but also will justify its the spirit of the initiative.

The sequencing is present in several areas of research. In the context of Adaptive Educational Hypermedia systems (AEH) applied to distance learning, sequencing of content should be carefully designed so that the learner does not get lost in hyperspace [16] [17]. The content to be presented to learners must be selected and adapted in terms of presentation and navigation [13] [15]. The sequencing of content has also a vital part in the Intelligent Tutoring Systems (ITS) [12] developed under the hypothesis that computer systems can model human learning in selecting the best scheduling learning strategy for each learner [14]. The objective of the majority of ITS is to adapt their training offer in a dynamic way, according to pedagogical rules that depend also on the reactions of the learner [8] [5].

In parallel with this research on sequencing, considerable effort has been devoted to developing standards to enable systems-based learning on the web to find, share, reuse, and export content in a standardized way such as the IMS [10], the ADL [2], the AICC [4], the ISO/IEC JTC1 SC36 [11], the IEEE/LTSC [7] and the W3C [25]. The function of the sequence was included in the specifications of the standard IMS Simple Sequencing [9] which is taken up by SCORM Sequencing and Navigation [3]. This feature provides the learner with the sequencing of content in order to guide $\mathrm{him} / \mathrm{her}$ in the learning space.

The three approaches STI, AEH and IMS SS are superficially similar. They are intended to provide not only an appropriate content to achieve the learning objective 
efficiently, but also have fundamental important differences. Indeed, the ITS and AEH use a model student and a model of concepts in learning to decide what content and what navigation structure to display, and how to present this content. The learner model can be initiated by previous knowledge and dynamically updated according to the behaviour of the learner. The model concepts described how information is structured and linked together using the concept of relationship. However, IMS SS specification describes three data models: Sequencing Definition Model that stores the rules of progression in the activities, the Tracking Model describes the results of the learner's interactions related to an objective, and Activity State Model which records the status of current activity. The learning model is implicit in the tracking model. It contains only the educational progress and does not support the features and preferences of the learner.

The aim of IMS SS is to ensure that the learner can perform all the activities that an author considers important, while avoiding unnecessary ones. The objective is to focus on the strategies of the author. On the other hand, the objective of ITS and $\mathrm{AEH}$ is to help the learner to be familiar with the complex space of relations between domain concepts to achieve the goals he has chosen. Thus, these systems are learner-centered.

The main objective of this paper is to describe our approach for modeling course sequencing based on graphs which is called a SMARTGraph pedagogic graph. This will help the author to well structure his/her course and pedagogic relations between the various pedagogic units which compose it and help the learner to be oriented during the browsing in the course.

Proposed approach for modeling the course sequencing

In our approach the teaching course/path was designed to allow each learner to express his/her capacities as well as possible, and to build assets in agreement with his/her pedagogical objectives. The course is composed of Learning Units (LU) which are interrelated by pedagogic relations [19]. It is up to the pedagogic sequencing to define these relations. It will have to determine which LU will be presented to the learner and when it will take place.

It must allow not only the conditional branch from one learning resource to other learning resources, according to whether the learner has carried out a certain stages or obtained a sufficient note, but also the fact that this last is permitted to subscribe in a LU. The pedagogic sequencing is the result of the application of composition techniques which can be, for example, the browsing of the tree structure in a linear way from a LU to another (Fig. 1). A more complex pedagogic sequencing can be based on the achievement of certain LUs, as prerequisite LUs, on the learner preferences or on the evaluation results.

SMARTGraph is a pedagogic graph which is included in this perspective of the pedagogic sequencing modelling. In SMARTGraph the nodes represent the learning units and the arcs (links) are the pedagogical constraints between pedagogical sequences. In order to express the pedagogic constraints, we use the prerequisites of formalism. A prerequisite of a LU is the set of the knowledge necessary to follow this LU in order to achieve a defined objective.

The prerequisite of LUs can be components of the same LU (it is the case of the chapters of the same course) or external LUs belonging to other LUs (it is the case of a chapter of another course or even of another cursus). Using this formalism, the notation used to express that the LUi is a prerequisite of the $\mathrm{LUj}$ is as follows: $\operatorname{Pr}(\mathrm{LUj})=\mathrm{LUi}$. The logical operators that allow us to express pedagogical constraints between different units of the course are:

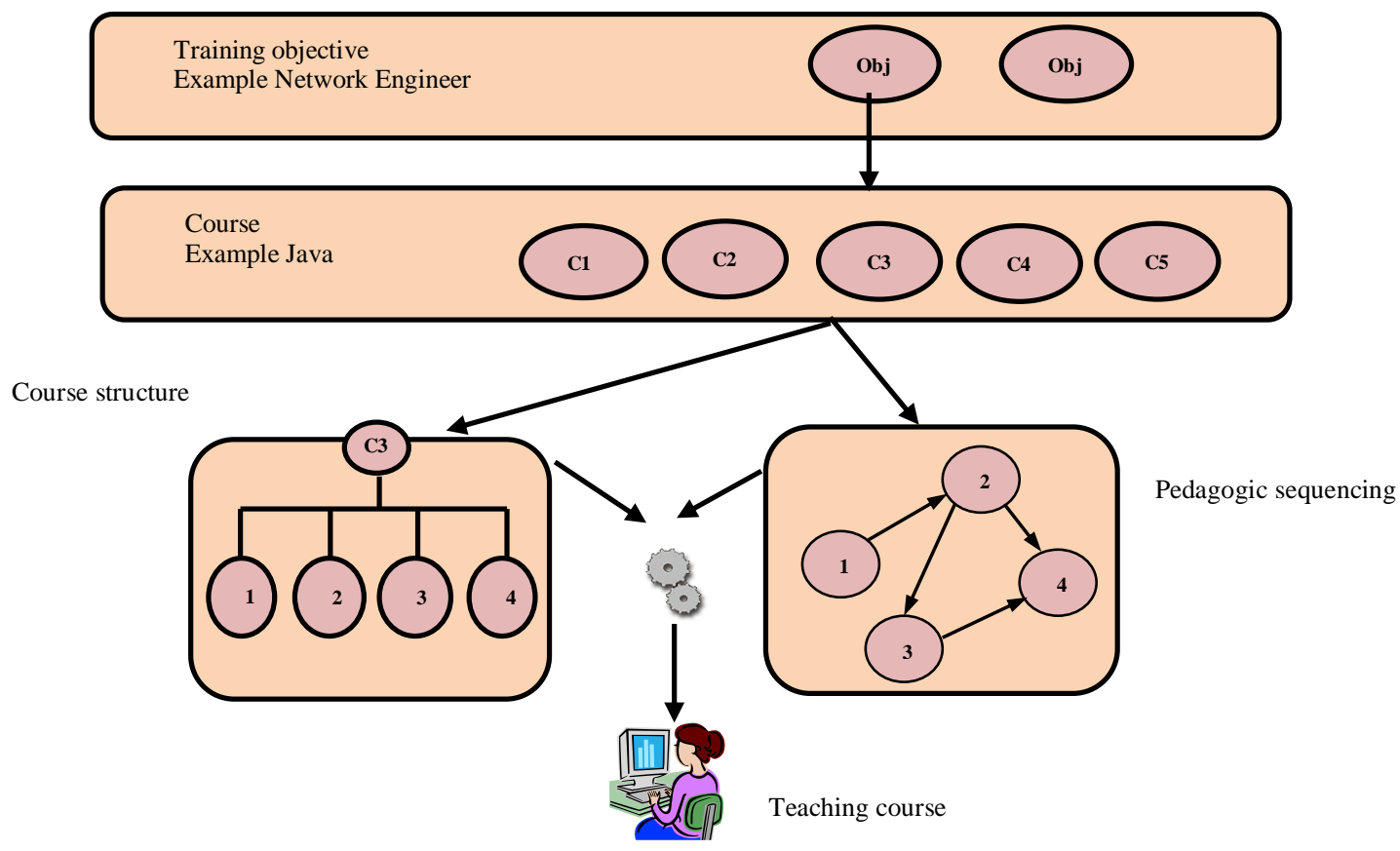

Figure 1. Proposed approach for course sequencing 


\section{A. No prerequisite}

$\operatorname{Pr}\left(L_{1}\right)=$ NULL. There is no LU required to follow $\mathrm{LU}_{1}$

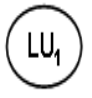

Figure 2. No prerequiste

\section{B. Simple prerequisite}

- Without condition: $\operatorname{Pr}(\mathrm{LU} 2)=\mathrm{LU} 1$. The learner can follow LU2 if he/she has followed LU1. It is equivalent to an unconditioned sequencing between two LUs of a graph.

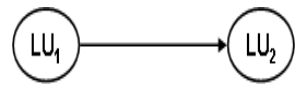

Figure 3. Simple prerequiste

- With condition: $\operatorname{Pr}($ LU2) = LU1 : c. The learner can follow LU2 if he/she has followed LU1 and satisfied the condition c. It is equivalent to a conditioned sequencing between two LUs. A condition $\mathrm{c}$ is boolean expression where the operators are $=,>,>=,<,<=$ and the operands are strings such as test score or number of attempts. To combine between multiple expressions we use the logical operators that are conjunction, disjunction and negation: $\&, \mid$ and !.

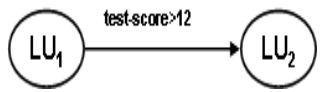

Figure 4. Prerequiste with condition

\section{Conjunction prerequisite}

- Without order: $\operatorname{Pr}(\mathrm{LU} 3)=\mathrm{LU} 1 \&$ LU2. The learner can follow LU3 if he/she has successfully followed LU1 and LU2.

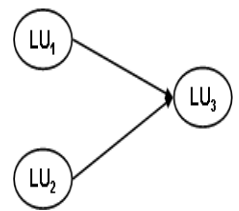

Figure 5. Conjonction prerequiste without

- With order $: \operatorname{Pr}(\mathrm{LU} 3)=\mathrm{LU} 1 \& \&$ LU2. The learner can follow LU3 if he/she has successfully followed LU1 and LU2 in the order.

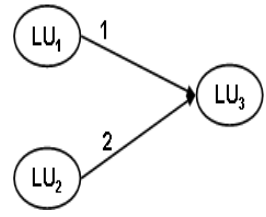

Figure 6. Conjonction prerequiste witht order

\section{Disjunction prerequisite}

- Simple: $\operatorname{Pr}(\mathrm{LU} 3)=\mathrm{LU} 1 \mid \mathrm{LU} 2 . \quad$ Choice between many LUs. The learner can follow either LU1 or LU2 to consider that the group LU1, LU2 has been done.

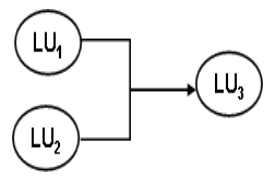

Figure 7. Disjonction prerequiste

- Choice of $n$ LU among m without any constraint on the followed order as prerequisite:

$\operatorname{Pr}(L U 4)=\{L U 1, L U 2, L U 3\} 2.2$ LUs of the set LU1, LU2, LU3, are sufficient to allow access to LU4.

- Choice of $n$ LU among $m$ with constraint on the followed order as prerequisite:

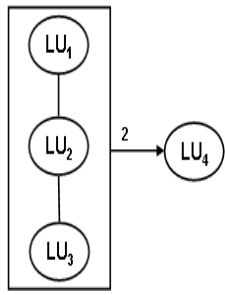

Figure 8: Choice 2 LUs among 3 without order

- $\operatorname{Pr}(\mathrm{LU} 5)=[\mathrm{LU} 1, \mathrm{LU} 2, \mathrm{LU} 3, \mathrm{LU} 4] 2$. Two LUs of the group are sufficient to consider that the group has been done, but the order must be respected.

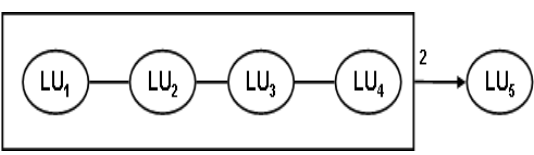

Figure 9. Choice 2 LUs among 4 witht order

E. Exclusive prerequisite

- $\operatorname{Pr}(\mathrm{LU} 3)=\mathrm{LU} 1^{\wedge} \mathrm{LU} 2$. The learner can follow either LU1 xor LU2 to consider that the group LU1, LU2 has been done.

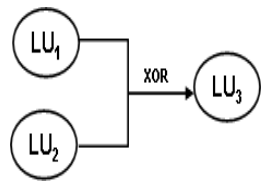

Figure 10. Exclusive prerequiste

In a complex expression of prerequisites we use parentheses to determine the precise order of evaluation of the expression. For example, on the expression $\operatorname{Pr}(\mathrm{LU} 4)=\mathrm{LU} 1 \&$ LU2| LU3, the learner can follow LU4 if he/she simply has followed LU3 or (LU1 and LU2). But if we add () like this $\operatorname{Pr}(\mathrm{LU} 4)=\mathrm{LU} 1 \&$ \& $(\mathrm{LU} 2 \mid \mathrm{LU} 3)$, the learner can follow LU4 if he/she has successfully completed LU2 or LU3 and the LU1.

Then, once the course is generated, the learner starts the first part, and at the end of this part, the system must take into account the learner's interactions during this learning unit. The learner will then have access to a specific learning unit if necessary with a modified profile. The transition from an educational unit to another is then made according to an educational approach. 


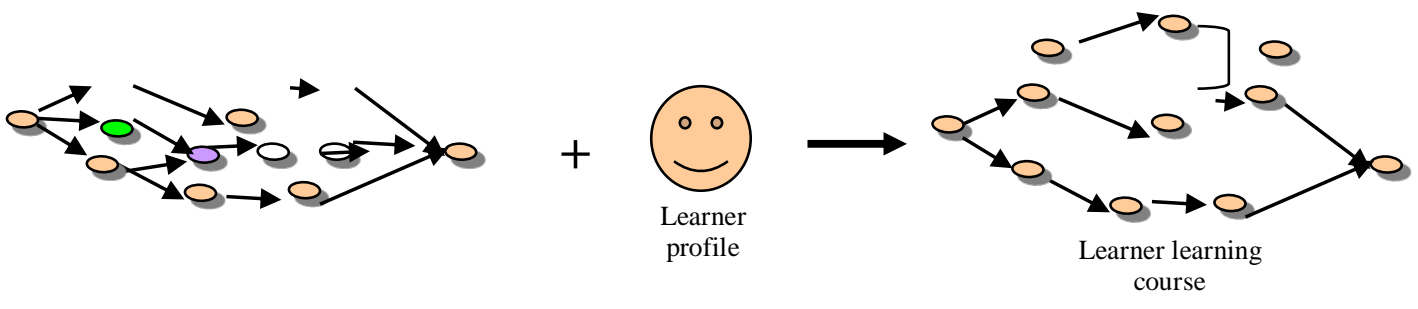

Figure 12. Personalization

The total graph is deduced starting from the prerequisites expressions because each definition of prerequisites makes it possible to deduce a portion from the pedagogical graph. This made, the fusion of the whole of under graph makes it possible to reconstitute the general graph.

The following scheme shows an example of SMARTGraph:
1) $\operatorname{Pr}\left(\mathrm{LU}_{1}\right)=\mathrm{NULL}$
2) $\operatorname{Pr}\left(\mathrm{LU}_{2}\right)=\mathrm{LU}_{1} \mid \mathrm{LU}_{2}: \mathrm{c}_{1}$
3) $\operatorname{Pr}\left(\mathrm{LU}_{3}\right)=\mathrm{LU}_{2} \& \mathrm{LU}_{6}$
4) $\operatorname{Pr}\left(L_{4}\right)=\mathrm{LU}_{3}$
5) $\operatorname{Pr}\left(\mathrm{LU}_{5}\right)=\mathrm{LU}_{3}$
6) $\operatorname{Pr}\left(\mathrm{LU}_{6}\right)=\mathrm{LU}_{1}$
7) $\operatorname{Pr}\left(\mathrm{LU}_{7}\right)=\mathrm{LU}_{6}: \mathrm{c}_{2}$
8) $\operatorname{Pr}\left(\mathrm{LU}_{8}\right)=\mathrm{LU}_{7} \&\left(\mathrm{LU}_{4} \mid \mathrm{LU}_{5}\right)$

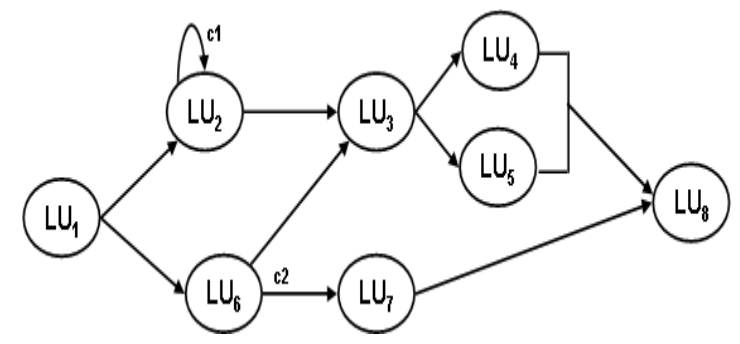

Figure 11. Example of SMARTGraph

While being based on the formalisms defined above, two approaches are offered to represent the pedagogical Graph: The first consists of representing the graph from the final or atomic elements of the hierarchical structure of the course. The second consists of breaking up the total graph into a hierarchy of under graph gathered by stage or level [18]. In this second case of figure, the course will be organized so that the passage of node to another is conditioned by the validation of under graph corresponding to this node. Contrary to the first representation, this one offers much more visibility and comprehension, because it makes it possible to preserve semantics on the regrouping of the course item.

\section{GRAPH ADAPTABILITY ACCORDING TO LEARNER PROFILE}

Thus the generation of the specific course sequencing consists of a simple extraction of a sub-graph of the general graph of the course (Fig. 12). The elements of the learners' profile will act here as a filter which lets pass only the pedagogical sequence with which they are in conformity [1].

The learner's profile is a key element in the implementation of adaptability in our approach. The profile is represented by an amount of information which characterizes the learner in the learning process. It consists in particular of the capacities, language, objectives of training and psychological factors of learning. Thus for the same learning objectives, each learner will have an adapted course, corresponding to his language, his learning speed or his capacities [20].

This requirement can be reached only by one good knowledge (modelling) of the learner. The purpose of the modelling of the learner is to establish the characteristics of learning depending in particular on his program, on his answers, on his preferences and his behavior to adapt the training to each individual; i.e. to define a profile for learning. This profile of learning can be subdivided into three components:

- The Intellectual profile which reflects the image of the learner on the educational level. The objective of this sub-model is to appreciate at every moment and with its right value, the state of knowledge of the learner.

- The criteria of localization which aim to facilitate the communication between the learner and the actors of the e-learning system and to allow a better comprehension of the course. We can quote such criterion of localization; the language, cultural origins and other criteria of localization.

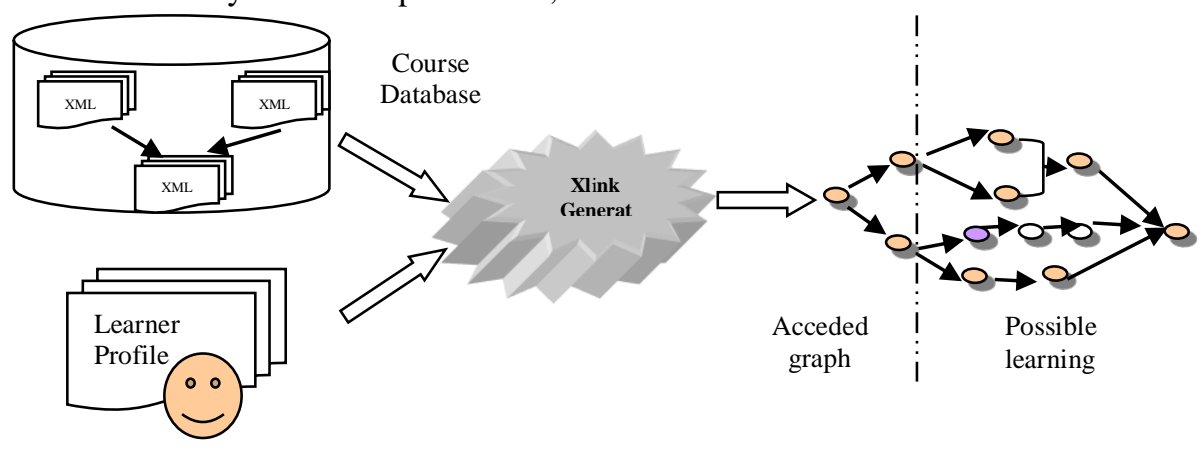

Figure 13: Implementation of SMARTGraph 
- A preference of the learner who represents these personal requirements so that the learner can evolve in an environment is familiar and convivial for him. For example, each learner will be able to visualize the course in the aesthetics of personalized colors.

\section{IMPLEMENTATION OF SEQUENCING}

Our objective is to provide an adaptive sequencing across pedagogical sequence a need for a good definition and structuring of the course documents is obvious for a comprehension of this structure and this definition by the system in order to extract some information targeted to provide course sequencing adapted to each learner's profile (Fig. 13).

In the search for a solution to our needs, our choice was made on XML [23] to describe the structure of the course, XLink (XML Linking Language) [24] to define the sequencing graph.

The adaptation of the course sequencing according to the user's profile is made possible using XSLT [22]. It will first of all be necessary to adopt a structure defining the relationship between the course and the profile.

The generation process consists of generating the already accomplished course by the learner in the first part of the graph and all the other possible courses in the second part. The continuation of the learner's course will be done according to his/her personal choices, and especially to his profile evolution.

\section{A. Technical choice: use of Xlink}

To satisfy our objectives for the implementation of the course sequencing, namely:

- The ability to know the paths followed by a learner during his progress in the course;

- Support for multi-directional links corresponding to an expression of combined pre-requisites (expression formulated from other expressions of prerequisites);

- To be able to manage the documents involved in the graph as LUs may be sources, target connection, or even both (i.e, a link with a series of locations and connections between them);

- And in the end to reach our main objective to have a sequencing document between learning units entirely separate from its contents.

Use of HTML hyperlinks has proven insufficient in our context. However, the XML linking technologies meets our needs perfectly. Indeed, XLink provides mechanisms rendering even more flexible hypermedia documents by:

- Extended Links: although richer than HTML links because they are multidirectional between multiple documents. An extended link is a directed graph in which the locations correspond to vertices and links between nodes correspond to arcs.

- Out-of-Line Links: such a link does not appear in the document or documents for which it constitutes a link.
This allows storing a series of definitions of links in a separate document which we called "basic links". Thus, we can define a document that can contain outof-line links that are totally separated from documents for which the links are active. This involves an intelligent separation between the document content and its sequencing. So we can change the order of the sequence of parts of a document without changing its contents; it suffices to modify its sequencing in the "basic links" document.

The notion of out-of-line extended links perfectly meets our afore-mentioned needs. We model the operators used in expressions of the prerequisites, linking the different learning sequences described in the pedagogical progression graph SMARTGraph; in arcs oriented more specifically in out-of-line extended links while taking account of the conditions of passage defined by the author.

In order to structure the elaboration of the implementation of SMARTGraph we proceed by decomposition in two stages: generation and adaptation.

\section{B. Generation process}

As shown in Fig. 14, starting from the list of the expressions of the prerequisites stored in the database, we generate generic XLINK corresponding to the total pedagogical graph of the Fig. 11 via an XSLT transformation.

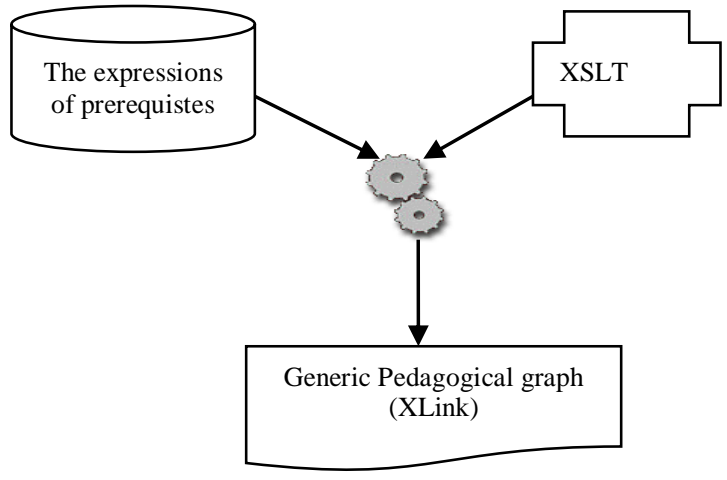

Figure 14. Pedagogical graph generation process

\section{Adaptation process}

As shown in Fig. 16, knowing that each learner has a profile which is brought to change constantly during all the learning process, it is unimaginable to envisage all possible XSLT transformations being able to be applied to the pedagogical graph.

This led us to choose the solution of a generic XSLT file containing a certain number of parameters which have a direct relationship with the profile (Fig. 17).

Thus, the parameter setting of this generic XSLT by the profile item will dynamically give a specific XSLT according to this profile (Fig. 18). The transformation of the generic XSLT into specific XSLT will be done by using XML parser such as DOM (Document Object Model) [21]. 


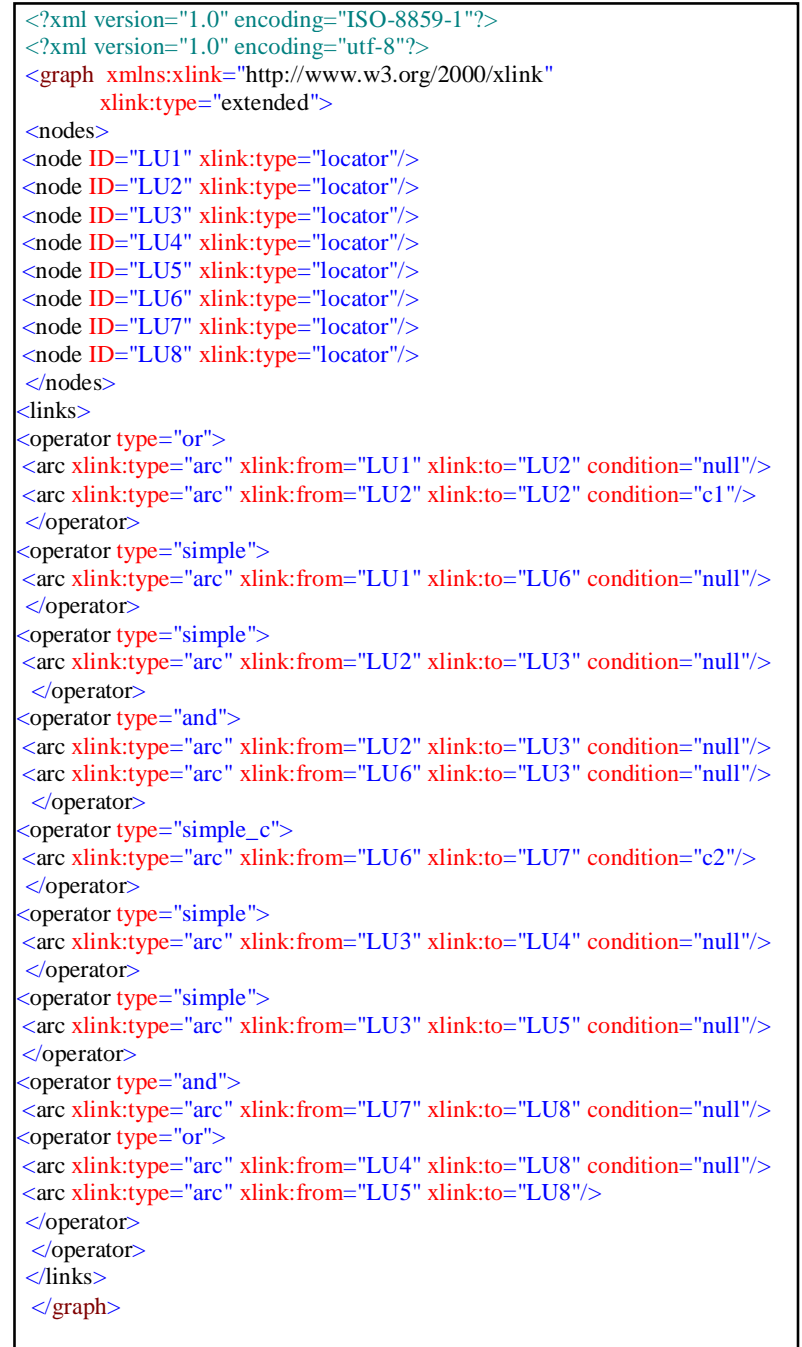

Figure 15 : Generic XLink file

TABLE I. PROFILE

\begin{tabular}{|c|l|}
\hline \multicolumn{2}{|c|}{ Profile } \\
\hline Pedagogical Objective & Engineer \\
\hline Accomplished units list & LU1,LU2, LU3, LU6 \\
\hline Language & French \\
\hline
\end{tabular}

<xsl:transform

xmlns:xsl="http://www.w3.org/1999/XSL/Transfor $\mathrm{m}$ " version $=" 1.0 ">$

$<$ xsl:variable name $=$ "Language" select $="$ "'"/>

$\langle$ xsl:variable name $=$ "List_LUs" select="'"/>

<xsl:variable name="Obj_Pedag" select="'"'/>

<xsl:template match="/">

$\langle$ xsl:for-each select="graph">

$<$ xsl:choose $>$

$\langle/ x s 1:$ choose $\rangle$

$</ x$ sl:for-each $>$

$</ x s 1:$ template $>$

$</ x$ sl:transform $>$
Applied to the values of the following profile:

Give the specific XSLT above:

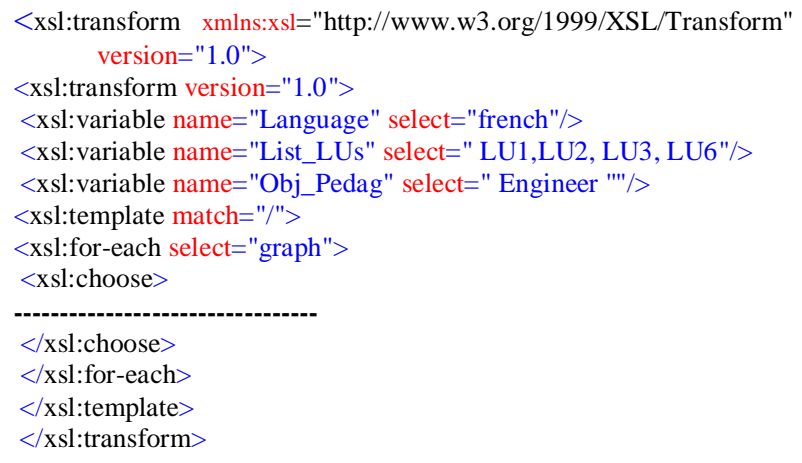

Figure 18 : Specific XSLT file

Thus, to generate the SMARTGraph specific to a profile, it is necessary to apply the generic pedagogical graph XLink XSL transformation specific to this profile.

Applied to the specific XSLT of Fig. 18 gives the specific pedagogical Graph below:

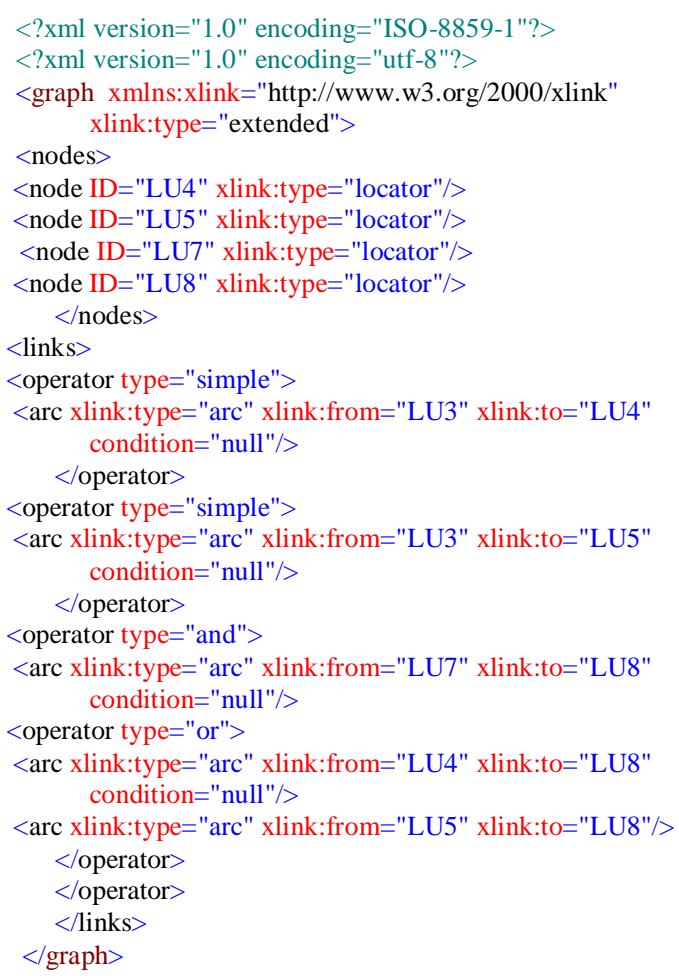

Figure 17 : Generic XSLT file 


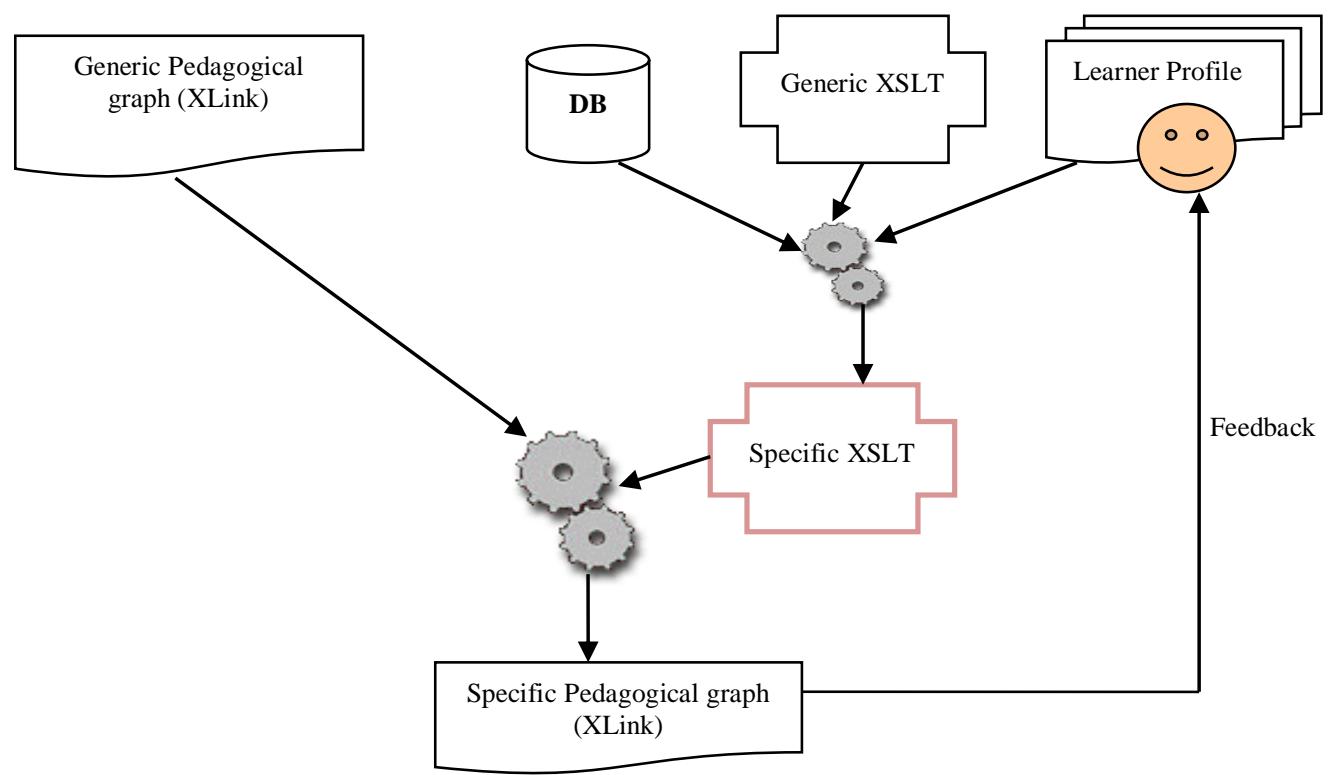

Figure 15. Adaptation process

\section{CONCLUSION}

In this paper we have proposed a modelling approach of course sequencing based on graphs. These graphs pedagogical in nature are a projection of the learning paths of the learner defined by the content designer as pedagogical constraints. In our approach the relationship of prerequisites is the translation of these pedagogical constraints. The combination of the prerequisites determines the educational graph SMARTGraph, hence modeling the possible sequencing within the educational content. The SMARTGraph nodes are the learning units and the arcs are the pedagogic constraints between these units.

SMARTGraph allows the real-time modelling of the choice and the succession of the courses, or the parts of a course that a learner operates during his/her training. This modelling consists of presenting the relations between the different parts of a course, or a cursus, by means of algebraic operators. These operators are used, in this sense, as constructors of the sequencing of the parts of a course for a learner's profile at a given time. In fact, this sequencing allows or does not specify access instructions according to the operators result.

The adaptability proposed by our model is not restricted only to the course content, but also to the sequencing of its content. This provides our approach with a high level of adaptability and flexibility.

Based on standard technologies such as XML, XLink and XSLT in our implementation system, we have proved the relevance of the concepts which we have presented.

\section{ACKNOWLEDGEMENT}

The authors wish to give their sincere thanks to Professor Ali BOUTOULOUT the director of the research group TSI (Théorie des Systèmes et Informatique) that allows us to conduct the researches.

\section{REFERENCES}

[1] A. Benkiran and R. Ajhoun "an Adaptive and cooperative telelearning system: SMART-Learning", International Journal on E-Learning AprilJune 2002, Vol.1, No.2 pp: 60-72.

[2] ADL Shareable Courseware Object Reference Model: SCORM Version 1.3, 2004. Available from http://www.adlnet.org/

[3] ADL, "SCORM Sequencing and Navigation (SN) Version 1.3", http://www.adlnet.gov, 2004.

[4] AICC, “Aviation industry CBT committees", http://www.aicc.org, 1998.

[5] C. J. Butz, S. Hua, and R. B. Maguire, "A web-based intelligent tutoring system for computer programming", In WI '04: Proc. Of the 2004 IEEE/WIC/ACM Int.Conf. on Web Intelligence. IEEE Computer Society, 2004.

[6] D. Bouzidi , R. Elouahabi and N. Abghour "SMARTNotes: Semantic annotation system for a collaborative learning", International Journal of Computer Science Issues, Vol. 8, Issue 6, No 1, November 2011.

[7] IEEE/LTSC, "IEEE Learning Technology Standers Committee", http://ieeeltsc.org/, 1997.

[8] G. Weber and P. Brusilovsky (2001). "ELM-ART: An adaptive versatile system for Web-based instruction", International Journal of Artificial Intelligence in Education, 12 (4), Special Issue on Adaptive and Intelligent Web-based Educational Systems, 351-384.

[9] IMS, "IMS Simple Sequencing Information and Behavior Model, Version $1.0 \quad$ Final $\quad$ Specification”, http://www.imsglobal.org/simplesequencing, 2003.

[10] IMS, "IMS Global Learning Consortium", http://www.imsglobal.org, 1997.

[11] ISO/IEC JTC1 SC36, "ISO/IEC JTC1 SC36 Standards for: information technology for Learning, Education, and Training", http://jtc1 sc36.org/index.html, 1999.

[12] P. Brusilovsky, and C. Peylo (2003), "Adaptive and Intelligent Webbased Educational Systems", International Journal of Artificial Intelligence in Education, 13 156-169.

[13] P. Brusilovsky, "Adaptive navigation support in educational hypermedia: The role of student knowledge level and the case for metaadaptation", British Journal of Educational Technology, 34(4):487-497, 2003. 
[14] P. Brusilovsky and J. Vassileva (2003), "Course sequencing techniques for large-scale Web-based education", International Journal of Continuing Engineering Education and Life-long Learning, Vol.13.

[15] P. Brusilowsky, "Adaptive Hypermedia" Journal of User Modeling and User-Adapted Interaction, Vol. 11, pp. 87-110, 2001.

[16] P. De Bra, G.J. Houben and H. Wu, "AHAM: A Dexter-based Reference Model for Adaptive Hypermedia", Proceedings of the 10th ACM Conference on Hypertext and Hypermedia, ACM Press, 1999, pp. 147156

[17] P. De Bra , D. Smits and N. Stash, "Creating and delivering adaptive courses with AHA!" In EC-TEL 2006, pp. 21-33

[18] R. Elouahbi A.Nassir and S.Benhlima, "Design and implementation of adaptive sequencing based on graphs", 11th International Educational Technology Conference, Turkey, 2011, p 233-238.

[19] R. Elouahbi, "Modelisation et mise en œuvre du sequencement dans le systeme de tele-enseignement SMART-Learning via le graphe pedagogique SMARTGraph", Thèse de Doctorat, Spécialité Informatique, Ecole Mohammadia d'Ingénieurs (EMI), Université Mohammed V-Agdal, Rabat, 2005.

[20] S. B. M. Jonas, R. Elouahbi and A. Benkiran, "Installation of the Adaptability in Course Sequencing via the SMARTGraph pedagogic Graph " proceedings of ECEL 2004 the The 3rd European Conference on e-Learning, Paris, France, November 2004, pp. 597-604.

[21] World Wide Web Consortium, Document Object Model DOM Level 2, http://www.w3.org/TR/DOM-Level-2-Core/.

[22] World Wide Web Consortium, eXtensible Stylesheet Language, XSL Specification version 1.0, W3C Recommendation, October 15, 2001. http://www.w3.org/TR/xsl/

[23] World Wide Web Consortium, Extensible Markup Language, XML Specification -version 1.0 (Second Edition), W3C Recommendation, October 6, 2000. http://www.w3.org/TR/2000/REC-xml-20001006

[24] World Wide Web Consortium, XML Linking Language (XLink) Version $\quad 1.1, \quad 06 \quad$ May 2010. http://www.w3.org/TR/2010/REC-xlink11-20100506/.
[25] W3C, "World Wide Web Consortium", http://www.w3.org, 1994.

\section{AUTHORS PROFILE}

Rachid ELOUAHBI received his doctorate degree in Computer Science from Mohammadia School of Engineering Morocco in 2005. He is currently an Assistant Professor at the University of Moulay Ismail, Meknès. His research focused on the area of adaptive learning systems, course sequencing and learning technologies. He works on the project Graduate's Insertion and Assessment as tools for Moroccan Higher Education Governance and Management in partnership with the European Union.

Driss BOUZIDI is an Assistance Professor in Computer Science at Hassan II Ain Chock University, Faculty of Sciences Casablanca, Morocco. He received a Ph.D. degree in computer engineering from Mohammed V University in 2004 on "Collaboration in hypermedia courses of e-learning system SMARTLearning". Dr. Bouzidi's research interests are in Distributed multimedia Applications, Collaboration systems, and elearning. He was vice-chair of the international conference NGNS'09 (Next Generation Network and Services) and the treasurer of the two research associations e-NGN and APRIMT.

Noreddine ABGHOUR is currently an Assistant Professor at the Faculty of Sciences Ain Chok, Casablanca, Department of Mathematics and Computer Science. $\mathrm{He}$ is also a $\mathrm{PhD}$ in Computer Science, area of distributed applications, from The Polytechnic Institute of Toulouse. In addition to teaching, his research interests are in the field of security and collaborative systems.

Mohammed Adil NASSIR is currently a PhD student at the Faculty of Sciences Meknes, Department of Mathematics and Computer Science. He is a Technical Engineer in Computer Systems and a specialist in local area networks from St-Petersburg Technical University - Russia. He works on the project of Graduate's Insertion and Assessment as tools for Moroccan Higher Education Governance and Management in partnership with the European Union. 